\title{
CRECIMIENTO DE PLÁNTULAS DE MAíz (Zea mays L. var. Porva) EN SOLUCIÓN NUTRITIVA CON BAJA RELACIÓN $(\mathrm{Ca}+\mathrm{Mg}+\mathrm{K}) / \mathrm{Al}$
}

\section{GROWTH OF MAIZE SEEDLINGS (Zea mays L. var. Porva) IN NUTRIENT SOLUTION WITH LOW $(\mathrm{Ca}+\mathrm{Mg}+\mathrm{K}) / \mathrm{Al}$ ratio}

\author{
Fánor Casierra-Posada1', Julián Cárdenas-Hernández²
}

\begin{abstract}
${ }^{1}$ Ingeniero Agrónomo Ph.D., docente asociado en la Facultad de Ciencias Agropecuarias, UPTC. Grupo de Investigación Ecofisiología Vegetal. Apartado aéreo 661, Tunja - Boyacá, e-mail: fanor.casierra@uptc.edu.co (Autor para correspondencia). ${ }^{2}$ Estudiante de Ingeniería agronómica, UPTC, Tunja. E-mail: julianc0182@yahoo.es
\end{abstract}

Rev. U.D.C.A Act. E Div. Cient. 12 (2): 91-100, 2009

\section{RESUMEN}

La toxicidad por aluminio, se relaciona en la productividad de cultivos establecidos en suelos ácidos con alta disponibilidad de $\mathrm{Al}^{3+}$. Frecuentemente, a valores de $\mathrm{pH}$ inferiores a 5,0, existe un efecto tóxico en especies agrícolas importantes, debido a la solubilidad del $\mathrm{Al}^{3+}$. Este trabajo, se llevó a cabo en Tunja, Boyacá, Colombia, para determinar el efecto tóxico del aluminio en el crecimiento de plántulas de maíz (Zea mays L. var. Porva). Las plántulas crecieron en solución nutritiva gaseosa (macro y micronutrientes), en relaciones $(\mathrm{Ca}+\mathrm{Mg}+\mathrm{K}) /$ $\mathrm{Al} 1 ; 0,75$ ó 0,5, mediante la adición de $\mathrm{Al}_{2}\left(\mathrm{SO}_{4}\right)_{3} \cdot 18 \mathrm{H}_{2} \mathrm{O}$ en relación con las bases ( $\mathrm{Ca}, \mathrm{Mg}$ y K), contenidas en la solución. Al medio de crecimiento de las plantas testigo, no se les adicionó aluminio. El pH, se ajustó a 5,5 para todos los tratamientos. Las plántulas fueron analizadas cuando se hicieron evidentes los síntomas por toxicidad de aluminio. Se estimó la longitud radical total, el peso seco y el área foliar; adicionalmente, se calculó la relación raíz/vástago. El área foliar, peso seco y longitud radical total no mostraron diferencias estadísticas entre los tratamientos con relaciones $(\mathrm{Ca}+\mathrm{Mg}+\mathrm{K}) / \mathrm{Al} 1 \mathrm{a}$ 0,5; sin embargo, todos los tratamientos con aluminio fueron estadísticamente diferentes al control. Los valores de la relación raíz/vástago mostraron una tendencia inversamente proporcional con la relación $(\mathrm{Ca}+\mathrm{Mg}+\mathrm{K}) /$ $\mathrm{Al}$; estos resultados implican que la variedad de maíz
Porva es muy sensible al aluminio, independientemente si la relación bases/aluminio se encuentre baja o en balance.

Palabras clave: Toxicidad, estrés, aluminio, suelos ácidos.

\section{SUMMARY}

Aluminium injury or toxicity is frequently related to field crops grown in acid soils with high $\mathrm{Al}^{3+}$ availability. Often, $\mathrm{pH}$ values below 5.0 have a toxic effect on the growth of agriculturally important plant species, because of the $\mathrm{Al}^{3+}$ solubility. An experiment was carried out in Tunja/ Colombia to determine the toxic effect of aluminium on the growth of maize seedlings (Zea mays L. var. Porva). Seedlings were grown in aerated nutrient solution (macro and micronutrients) containing $1,0.75$ or 0.5 $(\mathrm{Ca}+\mathrm{Mg}+\mathrm{K}) / \mathrm{Al}$ ratios by addition of $\mathrm{Al}_{2}\left(\mathrm{SO}_{4}\right)_{3} \cdot 18 \mathrm{H}_{2} \mathrm{O}$ in relation to the bases ( $\mathrm{Ca}, \mathrm{Mg}$ and $\mathrm{K}$ ) content in the solution. To the growth solution of the control plantlets no $\mathrm{Al}$ was added. The $\mathrm{pH}$ was adjusted to 5.5 in all treatments. Seedlings were harvested when the Al-toxicity symptoms were evident. The total root length, the dry matter production and the leaf area were measured; additionally, the root/shoot ratio was calculated. The leaf area, the total dry matter production and the total root length showed no statistical differences between 
treatments with 1 to $0.5(\mathrm{Ca}+\mathrm{Mg}+\mathrm{K}) / \mathrm{Al}$ ratios, but all treatments containing $\mathrm{Al}$ were statistically different to the control. The value of the root/shoot ratio showed an inversely proportional tendency to the $(\mathrm{Ca}+\mathrm{Mg}+\mathrm{K}) / \mathrm{Al}$ ratio. These results suggest that the maize variety Porva is very Al-sensitive, independently of a low or in balance bases/Al ratio.

Key words: Toxicity, stress, aluminum, acid soils.

\section{INTRODUCCIÓN}

Entre los cereales cultivados en el mundo, el maíz ocupa el primer lugar en términos de producción y, el tercero, después del trigo y el arroz, en relación a área cubierta (FAO, 2004). A nivel nacional, el maíz cultivado, tradicionalmente, ocupó un área de 478.721ha, en 2007, con un rendimiento de $1,6 \mathrm{t}_{\text {.ha }}{ }^{-1}$, mientras que el maíz tecnificado, se sembró en el mismo año, en 147.895ha, con rendimientos de 4,2t. ha-1 (Agronet, 2008), lo cual, es un referente de la relevancia que representa el cultivo para los agricultores colombianos. Pese a la superficie sembrada, el cultivo se desarrolla en algunas localidades con problemas de acidez intercambiable alta y riesgo de toxicidad por aluminio. En respuesta, se ha buscado el desarrollo de materiales de Zea mays que se ajusten a condiciones ácidas y con baja disponibilidad de nutrientes; tal es el caso de los híbridos de maíz Corpoica H-108 y Corpoica Altillanura H-111 (De León et al. 2000; 2001). La toxicidad por aluminio en suelos ácidos impacta negativamente la producción en plantas cultivadas y, en particular, en cereales.

Dado que, aproximadamente $30 \%$, de la superficie agrícola en el mundo está formada por suelos ácidos y el $50 \%$ de la superficie potencialmente arable es ácida, la toxicidad por aluminio representa una de las limitaciones más importantes en la producción agrícola en todo el mundo (Von Ulexküll \& Mutert, 1995). Alrededor de un $64 \%$ de los suelos tropicales de América son ácidos y su alta concentración de $\mathrm{Al}^{3+}$ es, con frecuencia, la razón del fracaso del cultivo de maíz (Sánchez, 1977). La superficie del suelo colombiano afectado por acidez, con valores de $\mathrm{pH}$ inferiores a 5,5 alcanza 67 millones de hectáreas, que corresponde al 58,8\% del territorio nacional (Arcos, 2008).

En cuanto a los cationes, mientras $\mathrm{Ca}, \mathrm{Mg}$ y K representan elementos esenciales para las plantas, el $\mathrm{Al}^{3+}$ es tóxico.
Su actividad en suelos ácidos depende de la forma en que se encuentre. Según el pH del suelo, el aluminio está desde las formas monoméricas $\mathrm{Al}^{3+}$ (soluble en agua) y $\mathrm{Al}(\mathrm{OH})$ (tóxico para las plantas), hasta las formas polimerizadas de hidróxidos de aluminio y de aluminatos. Se considera que el $\mathrm{Al}^{3+}$ es más tóxico para las plantas a pH 4,5 que a 4,0 y que la inhibición del crecimiento radicular, se debe a la forma hidrolítica $\mathrm{Al}(\mathrm{OH})$, más que a la forma monomérica $\mathrm{Al}^{3+}$. A pH entre 5,5 y 7,5, el $\mathrm{Al}^{3+}$, se encuentra precipitado (Salinas, 1988).

La toxicidad de aluminio es un problema bastante serio, generado como consecuencia de la acidificación de los suelos. Es por eso que los mecanismos de control de la solubilidad de $\mathrm{Al}^{3+}$, han sido uno de los tópicos importantes de investigación, especialmente, en las últimas décadas (Panda et al. 2003; Ma, 2005; CasierraPosada E Niño-Medina, 2007).

Ampliamente, se ha documentado la toxicidad por $\mathrm{Al}^{3+}$ como uno de los principales problemas en los suelos tropicales ácidos. Los suelos muy evolucionados, como los oxisoles y ultisoles, son naturalmente ácidos y algunas otras categorías, se pueden acidificar dependiendo de su uso y su manejo. En Colombia, la acidez del suelo constituye un problema de gran magnitud en suelos ubicados en las cordilleras andinas, en los valles interandinos de aluviones ácidos, en la Orinoquía, la Amazonía y en las demás regiones colombianas, caracterizadas por precipitaciones altas y frecuentes (Salinas, 1988; Espinosa, 2001).

Los cultivares de $Z$. mays tolerantes muestran diferentes mecanismos de toxicidad, con la participación de formas monoméricas o poliméricas de aluminio que, de alguna manera llegan, a la solución del suelo (Comin et al. 1999). El aluminio fácilmente se puede polimerizar y transformar de forma monomérica $\left(\mathrm{Al}^{+3}\right)$ a una forma polimérica $\left(\mathrm{Al}_{13}\right)$. Esta última es más fitotóxica para Z. mays (Bell \& Edwards, 1986). Adicionalmente, Rayburn et al. (1993) notificaron efectos a nivel de los ácidos nucleicos causados por $\mathrm{Al}^{+3}$ en la planta. Se encontró también variabilidad entre diferentes isolíneas de Triticum sp. en respuesta a aluminio (Wetzel et al. 1999), así como cuatro genes que amortiguan la toxicidad (Ezaki et al. 2001). Las respuestas comunes de genotipos tolerantes al exceso de aluminio, se presentan acorde con la capacidad de las plantas para modificar el pH del continuum suelo/raíz (Mengel E Kirkby, 1987; El-Shatnawi E Makhadmeh, 2001). La detoxificación 
de $\mathrm{Al}^{+3}$ mediante la quelatación, se puede desarrollar, a través de la excreción de ácidos orgánicos y polifenoles hacia la rizósfera (Kayama, 2001; Tesfaye et al. 2001), implicando eventualmente la alteración en los niveles de Mg y Ca (Silva et al. 2001; Yang et al. 2001).

El objetivo de este trabajo fue la evaluación del crecimiento en plántulas de maíz var. Porva inducidas por alta saturación relativa de $\mathrm{Al}^{+3}$ con respecto al total de bases, tomando en consideración diferentes valores de la relación $\mathrm{Ca}+\mathrm{Mg}+\mathrm{K} / \mathrm{Al}^{+3}$.

\section{MATERIALES Y MÉTODOS}

El estudio tuvo lugar en un cuarto de crecimiento, en la Facultad de Ciencias Agropecuarias de la Universidad Pedagógica y Tecnológica de Colombia en Tunja, Boyacá, Colombia, ubicada a 2690msnm. Las condiciones ambientales durante el desarrollo del ensayo fueron: temperatura promedio $21,8^{\circ} \mathrm{C}$, humedad relativa $81,2 \%$ y flujo fotónico fotosintético en promedio de 49,7 $\mu \mathrm{mol} . \mathrm{m}^{-2} . \mathrm{s}^{-1}$ proveniente de luz natural, a través de teja plástica. Como material vegetal, se utilizaron plántulas de maíz de la variedad Porva. Las semillas germinaron en arena previamente lavada con $\mathrm{HCl} 1 \mathrm{~N}$ y enjuagada con abundante agua destilada. Cuando las plántulas desarrollaron dos hojas, se trasladaron a contenedores plásticos con capacidad para 500mL, en grupos de cuatro plantas por contenedor.

En cada contenedor, se colocaron $400 \mathrm{~mL}$ de una solución nutritiva, con la siguiente composición (los valores mencionados, se adicionaron en $\left.\mathrm{g}^{\cdot \mathrm{L}^{-1}}\right)$ : $\mathrm{N}: 0,4$; $\mathrm{P}_{2} \mathrm{O}_{5}: 0,03 ; \mathrm{K}_{2} \mathrm{O}: 0,05 ; \mathrm{CaO}: 0,0005 ; \mathrm{MgO}: 0,0013$; $:$ 0,00137; B: 0,0002; Cu: 0,00014; Fe: 0,00012; Mn: 0,0013; Mo: 0,00005 y Zn: 0,0002. De acuerdo con la cantidad de calcio, de magnesio y de potasio contenidos en la solución, se adicionó una cantidad diferente de aluminio, de manera que se lograra una relación $(\mathrm{C} a+\mathrm{Mg}+\mathrm{K}) / \mathrm{Al}$, equivalente a $1 ; 0,75$ y 0,5 en cada contenedor. Esta relación es uno de los criterios para definir como tóxica, una concentración determinada de aluminio en la solución del suelo (ICA, 1992). Como control se empleó la solución nutritiva sin la adición de aluminio y como fuente de $\mathrm{Al}$ se utilizó $\mathrm{Al}_{2}\left(\mathrm{SO}_{4}\right)_{3} \cdot 18 \mathrm{H}_{2} \mathrm{O}$ (Riedel-de Haën ${ }^{\circledR}$, Alemania).

En los tratamientos, incluso el control, el $\mathrm{pH}$ de la solución se ajustó a 5,5, mediante la adición de $\mathrm{NaOH}$ o
$\mathrm{HCl} \mathrm{0,1} \mathrm{N.} \mathrm{El} \mathrm{criterio} \mathrm{para} \mathrm{ajustar} \mathrm{el} \mathrm{pH}$ de las soluciones a 5,5 fue que a partir de este valor, el aluminio empieza a causar problemas en el suelo y, por consiguiente, en las plantas. Adicionalmente, se consideró que en condiciones de campo, a partir de este valor de $\mathrm{pH}$, una proporción cada vez mayor del $\mathrm{Al}^{3+}$ total presente en los suelos se encuentra ya sea en la solución del suelo. En esta forma iónica, el $\mathrm{Al}^{3+}$ es biodisponible, puede ser absorbido por las raíces de las plantas y lograr ser dañino para su crecimiento. Los contenedores, se cubrieron con papel aluminio con el propósito de limitar el crecimiento de algas en la solución. Para evitar condiciones de hipoxia en la solución, ésta se aireó durante todo el transcurso del ensayo, con la ayuda de motores para acuario.

Las plantas, se sacaron de los contenedores cinco semanas luego del transplante. La longitud total de las raíces se midió seccionando cada una de las raíces de la planta y colocándolas, en forma lineal, sobre una placa de vidrio con papel milimetrado por debajo. Para visualizar mejor las raíces sobre la placa de vidrio éstas se tiñeron con solución de azul de metileno durante diez minutos y, luego, se enjuagaron con agua destilada. El peso seco de los tejidos vegetales, se determinó con la ayuda de una estufa calibrada a $70^{\circ} \mathrm{C}$ hasta peso constante. El área foliar, se midió mediante un analizador LI-COR® 3000A (LI-COR, USA); la acumulación de materia seca en raíces, mediante el secado a $70^{\circ} \mathrm{C}$ hasta peso constante y su posterior cálculo, con base en la materia seca total de la planta; la relación raíz/ vástago, como el cociente del peso seco de la raíz y el de la parte aérea.

Se utilizó un diseño estadístico, completamente al azar, con cuatro replicaciones por tratamiento y una plántula, como unidad de muestreo. Los resultados obtenidos, se sometieron a un análisis clásico de variancia, mediante una tabla ANAVA. La diferencia entre promedios, se calculó con la prueba Tukey $(P<0,05)$; los análisis estadísticos, se realizaron con la versión 11.5.1 de SPSS (B) (Statistical Product and Service Solutions, Chicago, Illinois, USA - Nov. 2002). Las figuras, se presentan en formato de barras con su respectiva desviación estándar y el resultado de la prueba de Tukey.

\section{RESULTADOS Y DISCUSIÓN}

Área foliar: El comportamiento de las plántulas de maíz, en cuanto al área foliar, arrojó diferencias altamente 
significativas $(P<0,01)$. Fue así, como se presentaron diferencias entre los tratamientos implementados con el control, pero no entre los tratamientos que contenían aluminio. La proporción de aluminio con respecto a las bases en las solución nutritiva redujo el área foliar en términos de 38,6; 39,1 y 41,7\% para las relaciones $(\mathrm{Ca}+\mathrm{Mg}+\mathrm{K}) / \mathrm{Al}$ equivalentes a $1 ; 0,75$ y 0,5 , respectivamente, en relación con el control sin la adición de aluminio (Figura 1). El coeficiente de variación de Pearson para los tratamientos evaluados con respecto a esta variable fue de $29,5 \%$.

En contraposición a lo encontrado en el presente trabajo, en plantas de Betuna pendula, concentraciones menores de 5 ppm de $\mathrm{Al}$, incrementaron significativamente la expansión foliar, mientras que concentraciones por encima de 25ppm produjeron un efecto contrario en las hojas (Kidd \& Proctor, 2000). De igual manera, Symeonidis et al. (2003) encontraron una reducción, estadísticamente significativa, en el área foliar, equivalente a $52 \%$ en plantas de Cucumis melo expuestas a $\mathrm{Al}^{+3}$, en comparación con plantas testigo sin la presencia del elemento, lo cual, es un índice de la toxicidad producida, como consecuencia de la liberación de los protones, en presencia de $\mathrm{Al}^{+3}$ (Andersson $\&$ Brunet, 1993; Kinraide, 1993, 1997; Llugany et al. 1995); sin embargo, el hecho de que se haya obtenido resultados contradictorios en diferentes ensayos sugiere que el genotipo del vegetal está implicado en la capacidad de respuesta a la exposición a $\mathrm{Al}^{+3}$.

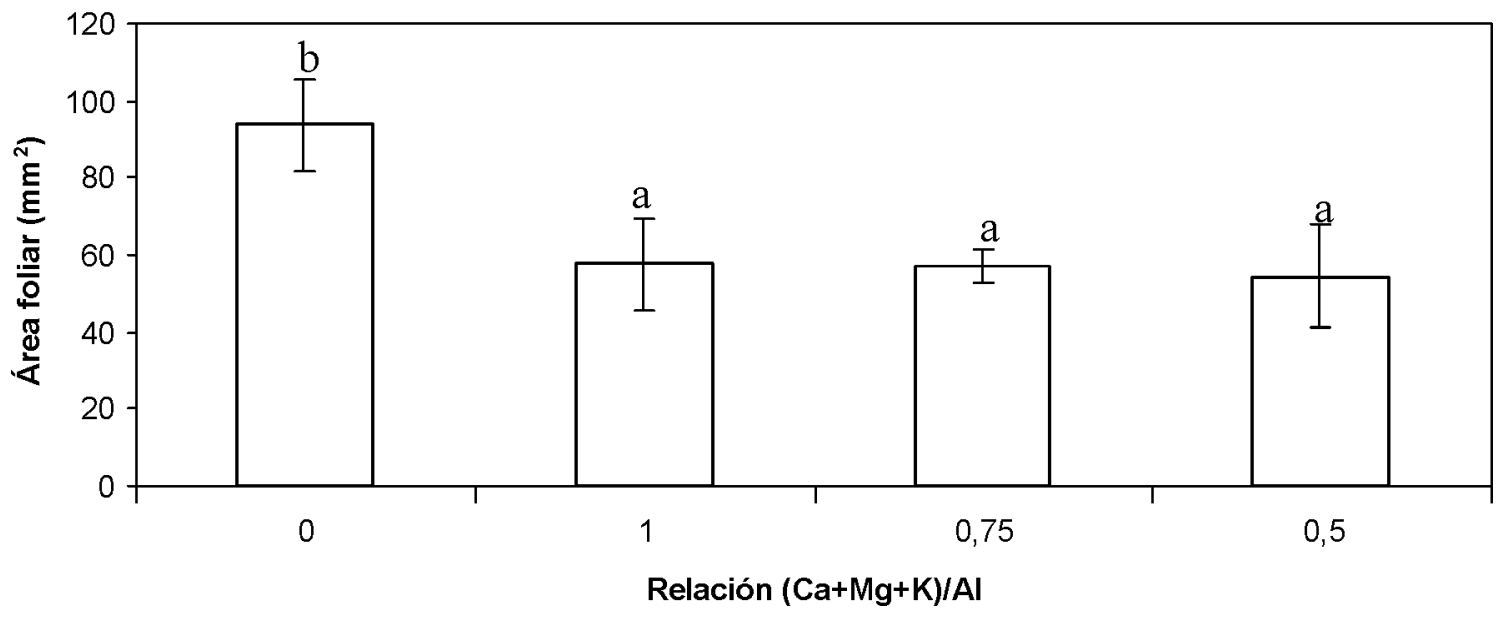

Figura 1. Área foliar promedio en plántulas de maíz (Z. mays L. var. Porva), afectadas por toxicidad por aluminio en solución nutritiva.

Al respecto, Konishi et al. (1985) observaron hojas más grandes en plantas de Camellia sinensis, una planta acumuladora de aluminio, cuando las plantas crecían en soluciones nutritivas suplementadas con contenidos de aluminio por encima de 172,7ppm. Por el contrario, Janhunen et al. (1995) encontraron una reducción en el tamaño y un incremento en la densidad de las agujas de pino Pinus sylvestris y Picea abies, luego de la exposición de las plantas a concentraciones elevadas de $\mathrm{Al}^{+3}$ (150ppm), con lo que se puede confirmar que las respuestas de los vegetales expuestos a $\mathrm{Al}^{+3}$ dependen ampliamente del genotipo.
Existen evidencias que el efecto positivo de la presencia de $\mathrm{Al}^{+3}$ sobre la promoción del crecimiento podría ser indirecto, debido a que el metal corregiría los efectos negativos ocasionados por otros elementos, como el cobre, que se encuentran en concentraciones elevadas y, con los cuales, el aluminio podría interactuar (Asher, 1991; Casierra-Posada, 2001). Se ha encontrado que el crecimiento de brotes y de raíces en Arachis hypogea se estimuló mediante la adición de $\mathrm{Al}^{+3}$ al sustrato, debido a que el metal redujo la toma de zinc y, por tanto, su acumulación en el vástago (Marschner, 1995). 
Peso seco total: Por otra parte, el peso seco total de las plántulas de maíz se vio afectado, significativamente, por los tratamientos $(P<0,05)$, apreciándose que la sola aplicación de $\mathrm{Al}^{+3}$ redujo considerablemente esta variable, en $50 \%$ para la relación $(\mathrm{Ca}+\mathrm{Mg}+\mathrm{K}) / \mathrm{Al} 1$ y 0,75 , mientras que con la relación de 0,5 , la reducción fue de 54,2\% (Figura 2). Es de anotar, que sólo se presentaron diferencias entre los tratamientos que contenían aluminio con el control, pero no entre los tratamientos con $\mathrm{Al}^{+3}$. El coeficiente de variación de Pearson en los tratamientos fue de $44,8 \%$ para este parámetro.

De igual manera, con lo reportado en el presente estudio, Symeonidis et al. (2003) encontraron una reducción en la acumulación de materia seca en plantas de C. melo, de manera inversamente proporcional al contenido de $\mathrm{Al}^{+3}$ en el sustrato. A pesar que el aluminio en concentraciones bajas provocó un incremento en la acumulación de materia seca en la medida en que se incrementó la concentración del elemento, las plantas manifestaron una reducción en la materia seca en comparación con las plantas control. Este efecto, puede ser atribuido a la disminución en la toxicidad de los protones exhibida por las plantas de C. melo en presencia de $\mathrm{Al}^{+3}$ (Andersson $\&$ Brunet, 1993; Kinraide, 1993, 1997; Osaki et al. 1997).

Adicionalmente, la estimulación del crecimiento y, por tanto, el incremento en la materia seca en las plantas, se puede basar en efectos específicos relacionados con la química compleja del aluminio en solución, mediante la cual, el elemento podría tener un efecto sobre la biodisponibilidad de otros iones en tal sentido que estimularía el crecimiento de las plantas (Clune $\mathcal{E}$ Copeland, 1999), como ya se había explicado.

El aluminio puede modificar los procesos metabólicos en los que participa el $\mathrm{Ca}^{2+}$, manteniendo niveles citoplasmáticos del elemento por encima de los valores normales o también mediante la prevención de la formación de tipos transitorios de Ca citoplasmáticos. De esta manera, muchos procesos metabólicos, que incluyen la multiplicación y la elongación celular se ven alterados (Bennet E Breen, 1991; Rengel, 1992; Ryan et al. 1993).

Longitud radical total: Presentó diferencias altamente significativas $(P<0,01)$; la proporción de $\mathrm{Al}^{+3}$, respecto a las bases en la solución nutritiva, redujo la longitud de las raíces en 73,7; 76,1 y 76,6\%, respectivamente, con las relaciones $(\mathrm{Ca}+\mathrm{Mg}+\mathrm{K}) / \mathrm{Al} 1 ; 0,75$ y 0,5. Para esta variable hubo $81,8 \%$ de dispersión calculada por el método de Pearson (Figura 3). La inhibición en la elongación radicular es uno de los síntomas más evidentes y de más rápida manifestación, observada en término de pocas horas o, incluso, minutos de exposición a $\mathrm{Al}^{+3}$ (Zhang $\mathcal{E}$ Rengel, 1999).

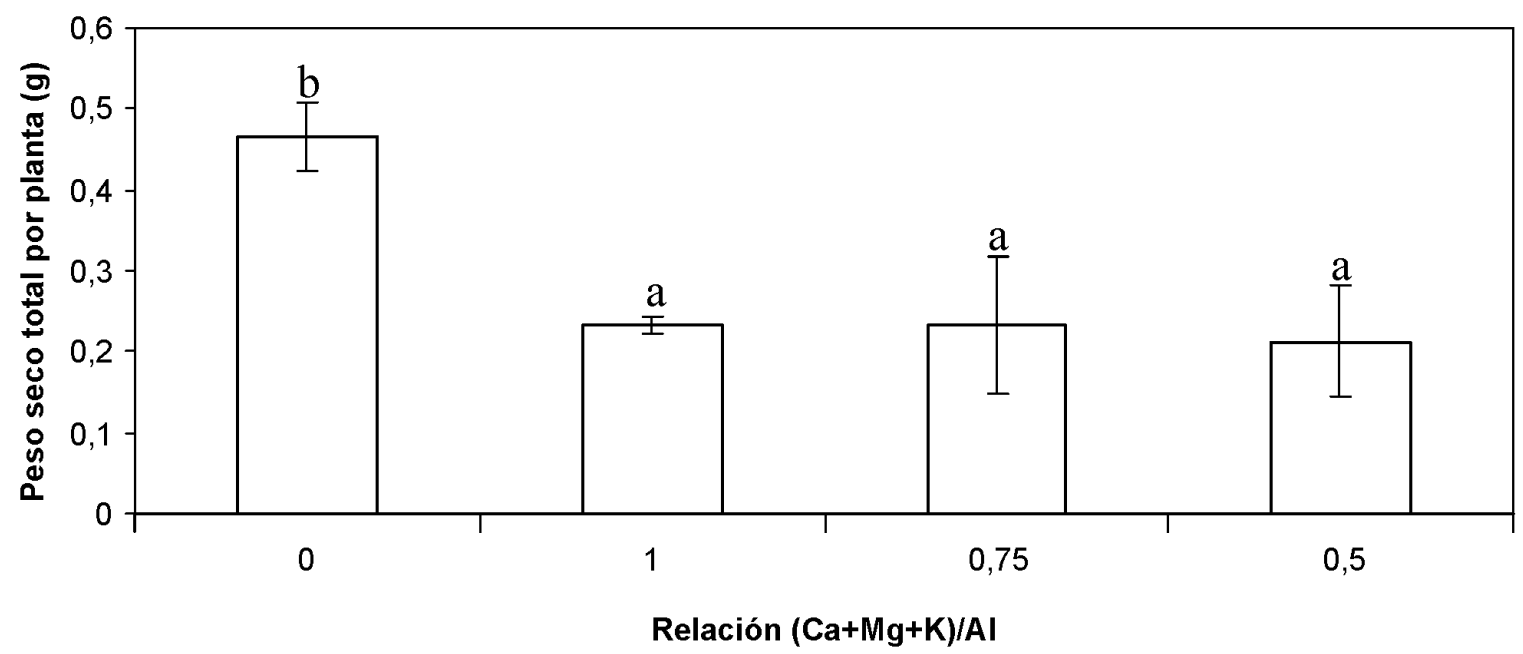

Figura 2. Peso seco total promedio en plántulas de maíz (Z. mays L. var. Porva), afectadas por toxicidad por aluminio en solución nutritiva. 


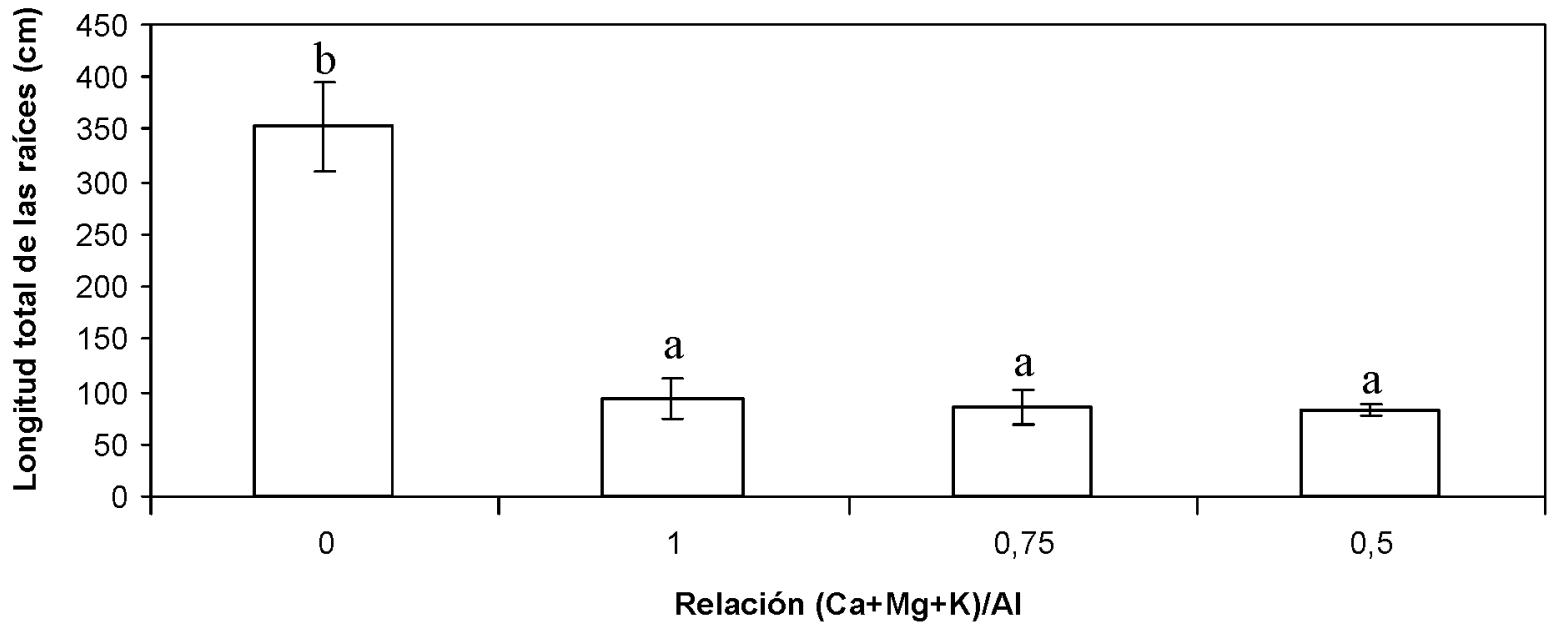

Figura 3. Longitud radical total promedio en plántulas de maíz (Z. mays L. var. Porva), afectadas por toxicidad por aluminio en solución nutritiva.

Independientemente de como el aluminio se libere y llegue a la solución del suelo, tanto las concentraciones altas como su proporción porcentual con respecto a la sumatoria de las bases $(\mathrm{Ca}+\mathrm{Mg}+\mathrm{K})$, posiblemente, tengan un efecto negativo de la reducción en la mitosis de meristemos radicales, debido a alteraciones inducidas por $\mathrm{Al}^{+3}$ en las moléculas del material genético (Matsumoto, 1991) y, en general, en el núcleo de las células meristemáticas. Se ha encontrado que en genotipos de vegetales sensibles a aluminio, éste se acumula en mayor cantidad y con más rapidez que en materiales tolerantes, aunque también existe la posibilidad que los materiales tolerantes limiten la entrada del aluminio a las raíces (Silva et al. 2000).

Los desórdenes causados, tanto en el núcleo de las células como en el material genético, tendrían como consecuencia alteraciones en la mitosis, pues es indiscutible el papel protagónico que los ácidos nucleicos y el núcleo tienen en los ciclos de división celular. Pero, si se tiene en cuenta que los ciclos celulares en los meristemos de las raíces duran entre 18 y 24 horas (Gunning \& Steer, 1996) es probable que la reducción de la división celular sea la responsable de la inhibición tan rápida del crecimiento radical, efecto que se expresa a partir de la primera hora de exposición de las raíces a aluminio. Una inhibición sustancial en el crecimiento de las raíces, que se extiende a lo largo de horas e incluso días, estaría asociada con la reducción de la multiplicación celular (Lazof E Holland, 1999).
La incorporación de $\mathrm{Al}^{+3}$ al material genético no es la única posible causal de la inhibición en la elongación radical, pues se ha reportado el papel de otros factores involucrados en este efecto; es el caso de la inhibición en el transporte de auxinas (Kollmeier et al. 2000) y la ruptura en la homeostasis citosólica del $\mathrm{Ca}^{2+}$ (Zhang $\mathcal{E}$ Rengel, 1999).

Si se asume que cuando la relación $(\mathrm{Ca}+\mathrm{Mg}+\mathrm{K}) / \mathrm{Al}$ es equivalente a la unidad, la toxicidad por $\mathrm{Al}$ sería contrarrestada con la concentración disponible de bases, lo cual, no resulta ser del todo cierto, desde el punto de vista biológico, puesto que, según la metodología implementada en el presente trabajo, la respuesta de las plantas en cuanto al crecimiento total de las raíces no manifestó deferencias, estadísticamente significativas, cuando la relación bases/aluminio estuvo en el rango entre 1 y 0,5 (Figura 3). Este resultado sugiere que a rangos de $\mathrm{pH}$ fuertemente ácidos, la sola presencia de $\mathrm{Al}^{+3}$ puede afectar, drásticamente, el crecimiento de las raíces, independientemente del contenido de bases disponibles en la solución del suelo. Esta apreciación difiere de lo encontrado por Casierra-Posada E Cárdenas-Hernández (2007), quienes aplicaron la misma metodología e igual valor de $\mathrm{pH}$ en la solución, en plántulas de Brassica oleracea var. Botrytis, hallando una reducción gradual en la longitud radical total de las plantas en proporción directa al valor de la relación $(\mathrm{Ca}+\mathrm{Mg}+\mathrm{K}) / \mathrm{Al}$. Detectaron diferencias, altamente significativas $(P<0,01)$, entre los tratamientos con 
relación bases $/ \mathrm{Al}^{+3}$ equivalente a uno hasta una relación equivalente a 0,5. En el presente trabajo, en el mismo rango de la relación bases $/ \mathrm{Al}^{+3}$, no hubo diferencias, estadísticamente significativas, en cuanto a la longitud radical total en plántulas de maíz, lo que indica una alta sensibilidad de la variedad Porva a la presencia de $\mathrm{Al}^{3+}$ en el sustrato en que crecen las plantas.
Relación raíz/vástago: Respecto a esta relación el análisis de variancia mostró diferencias significativas $(P<0,05)$. De esta forma, la adición de aluminio a la solución nutritiva incrementó los valores para este parámetro, en 10,$7 ; 24,9$ y 59,2\%, respectivamente, con la relación 1; 0,75 y 0,5 $(\mathrm{Ca}+\mathrm{Mg}+\mathrm{K}) / \mathrm{Al}$. La dispersión calculada por el método de Pearson fue de $24,2 \%$ para esta variable (Figura 4).

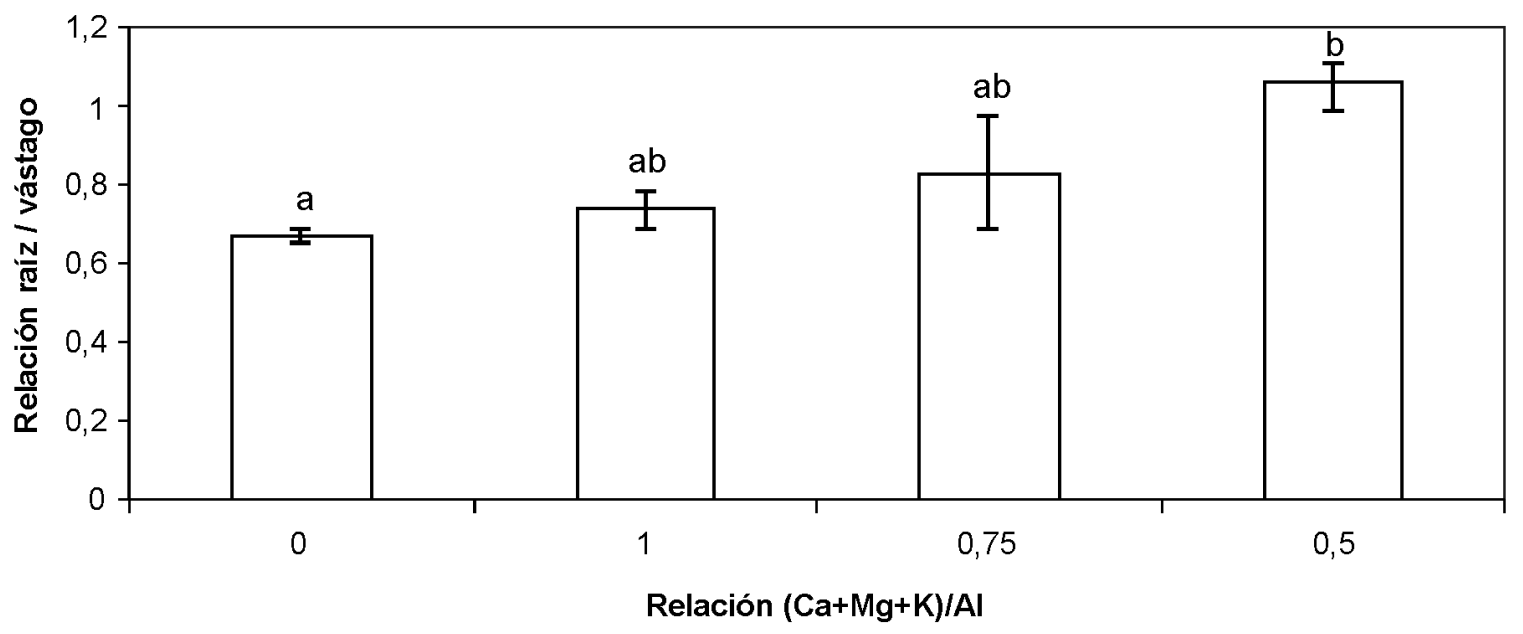

Figura 4. Relación raíz/vástago promedio en plántulas de maíz (Z. mays L. var. Porva), afectadas por toxicidad por aluminio en solución nutritiva.

Debido a que la relación raíz/vástago hace referencia al cociente entre el peso seco de la raíz y el peso seco de los órganos que conforman el dosel, una ganancia en peso en cualquiera de los factores tiene como consecuencia un desbalance de la misma. Por tanto, en el caso del presente ensayo, el comportamiento inversamente proporcional en el valor de la relación en cuestión, con respecto a la reducción en la relación evaluada $(\mathrm{Ca}+\mathrm{Mg}+\mathrm{K} / \mathrm{Al})$, se debió al efecto inhibitorio del $\mathrm{Al}^{3+}$, en cuanto al crecimiento de las raíces, el que provocó la reducción proporcional en la masa seca de las raíces induciendo un incremento en la relación raíz/vástago.

Como ya se discutió, son muchos los factores involucrados en el crecimiento radical (Casierra-Posada, 2002). Adicionalmente, se ha encontrado en células radicales de $Z$. mays, que la zona de elongación es el sitio donde la toxicidad por aluminio tiene lugar. Además, el aluminio induce la reorganización de los microtúbulos en la corteza interna; a pesar de lo cual, la orientación de los microtúbulos en la corteza externa y en la epidermis no sufre modificaciones, aún cuando los síntomas de toxicidad crónica ya se hayan manifestado. El tratamiento previo con aluminio en las raíces, inhibe la reorientación y la depolimerización de los microtúbulos inducidos por las auxinas y por el frío, respectivamente, lo cual, hace pensar que el aluminio provoca un incremento en la estabilidad de los microtúbulos en las células estudiadas. El efecto estabilizante provocado por el aluminio en las células de la corteza externa coincidió con la inhibición del crecimiento. En conclusión, la reorganización y la estabilización del citoesqueleto, están íntimamente ligadas a la toxicidad por aluminio, en raíces de $Z$. mays (Blancaflor et al. 1998) y, por tanto, en la inhibición de su crecimiento radical (Casierra-Posada, 2001; 2002). En el presente trabajo, así como en muchos otros ensayos realizados en vegetales, el efecto más evidente de una toxicidad por exceso de $\mathrm{Al}^{3+}$ en plantas de maíz, fue la reducción en el crecimiento de la raíz, en la cual, están implicados muchos factores. A pesar que se ha 
dado por hecho que el equilibrio en la relación bases/Al puede contribuir a mitigar los problemas de toxicidad por el metal, en la variedad de maíz estudiada y bajo las condiciones de hidroponía, la sola presencia del elemento en la solución nutritiva, independientemente del valor de la relación bases/Al, fue suficiente para que se presentaran los síntomas de toxicidad.

Conflictos de intereses: El manuscrito fue preparado y revisado con la participación de todos los autores, quienes declaramos que no existe ningún conflicto de intereses que ponga en riesgo la validez de los resultados presentados.

Financiación: Este estudio, se desarrolló con el apoyo financiero y logístico de la Dirección de Investigaciones (DIN) de la Universidad Pedagógica y Tecnológica de Colombia, en el marco del plan de trabajo del grupo de investigación Ecofisiología Vegetal, adscrito al programa de Ingeniería Agronómica de la Facultad de Ciencias Agropecuarias.

\section{BIBLIOGRAFÍA}

1. AGRONET. 2008. Área cosechada, producción y rendimiento de maíz tradicional, 1987-2007. Disponible desde Internet en: http:/www.agronet.gov.co/www/ htm3b/excepcionesNuke/cargaNet/netcarga16.as px?cod $=16$ Esubmit $=$ Ver + ReporteEreporte $=$ Pro ducci\%u00f3n+nacional + por + productoEfile $=2$ 007816102236_20058417048_agronetevaarea rendimientoyproduccionporproductoporagno.rp $\mathrm{t} E$ codigo $=16 \varepsilon$ excepcion $=1$ EfechaI $=1987 \varepsilon$ pro ducto $=$ Ma\%u00edz + tradicionalEfechaF $=2007$ (con acceso 18/11/08).

2. ANDERSSON, M.E.; BRUNET, J. 1993. Sensitivity to $\mathrm{H}$ - and $\mathrm{Al}$-ions limiting growth and distribution of the woodland grass Bromus benekennii. Plant Soil. 153:243-254.

3. ARCOS, A.L. 2008. Determinación de calosa para seleccionar genotipos de maíz resistentes a aluminio. Disponible desde Internet en: http://www. ciat.cgiar.org/training/pdf/2008_05_21_L_Arcos. pdf (con acceso 18/11/2008).

4. ASHER, C.J. 1991. Benefical elements, functional nutrients and possible new essential elements. En:
Mortvedt, F.; Cox, R.; Shuman, L.M.; Welch, R.M. eds. Micronutrients in agriculture. Soil Sci. Soc. Am. 4:703-723.

5. BELL, L.C.; EDWARDS, D.G. 1986. The role of aluminium in acid soil infertility. En: Latham, $M$. ed. Soil management under humid conditions, Bangkok, Thailand, p.201-223.

6. BENNET, R.J.; BREEN, C.M. 1991. The aluminium signal: New dimensions to mechanisms of aluminium tolerance. Plant Soil. 149:87-94.

7. BLANCAFLOR, E.B.; JONES, D.L.; GILROY, S. 1998. Alterations in the cytoskeleton accompany aluminum-induced growth inhibition and morphological changes in primary roots of maize. Plant Physiol. 118:159-172.

8. CASIERRA-POSADA, F. 2001. Fundamentos fisiológicos, bioquímicos y anatómicos del estrés por aluminio en vegetales. Rev. Comalfi. 28(2):8-19.

9. CASIERRA-POSADA, F. 2002. Alteraciones inducidas por aluminio en el citoesqueleto de las plantas. Rev. Comalfi. 29(2):23-28.

10. CASIERRA-POSADA, F.; CÁRDENAS-HERNÁNDEZ, J.F. 2007. Influencia del aluminio sobre el crecimiento de la raíz en coliflor (Brassica oleracea L. var. Botrytis, Hib. Nevada F1). Rev. U.D.C.A Act. E Div. Cient. 10(1):149-157.

11. CASIERRA-POSADA, F.; NIÑO-MEDINA, R.C. 2007. Solubilidad y reacción del aluminio en el suelo. Ciencia y Agricultura 5(2):7-17.

12. CLUNE, T.S.; COPELAND, L. 1999. Effects of aluminium on canola roots. Plant Soil. 216: 27-33.

13. COMIN, J.J.; BARLOY, J.; BOURRIÉ, J.; TROLARD, F. 1999. Differential effects of monomeric and polymeric aluminium on the root growth and on the biomass production of root and shoot of corn in solution culture. Eur. J. Agron. 11:115-122.

14. De LEÓN, C.; NARRO, L.; TORRES, L.G. 2000. Corpoica H-108; primer híbrido de maíz en Colombia para suelos ácidos de la Altillanura Plana. Villavicencio, Corpoica. Plegable divulgativo No. 17. 2p. 
15. De LEÓN, C.; NARRO, L.; TORRES, L.G.; Caicedo, S. 2001. Híbrido de maíz amarillo Corpoica Altillanura H-111. Villavicencio, Corpoica. Plegable divulgativo No. 2. 5p.

16. El-SHATNAWI, M.K.J.; MAKHADMEH, I.M. 2001. Ecophysiology of the plant-rhizosphere system. J. Agron. Crop Sci. 187:1-9.

17. ESPINOSA, J. 2001. Acidez y encalado de los suelos. En: Silva M., F., ed. Fertilidad de suelos: Diagnóstico y control. Soc. Col. Ciencia Suelo. p.113-128.

18. EZAKI, B.; KATSUHARA, M.; KAWAMURA, M.; MATSUMOTO, H. 2001. Different mechanisms of four aluminum (Al)-resistant transgenes for Al toxicity in Arabidopsis. Plant Physiol. 127:918-927.

19. FAO. 2004. Faostat. Disponible desde Internet en: http://faostat.fao.org/site/567/DesktopDefault. aspx?PageID=567. (con acceso 09/03/08).

20. GUNNING, B.E.S.; STEER, M.W. 1996. Plant cell biology: Structure and function. Jones and Bartlett ediciones, Sudbury, MA. 130p.

21. ICA INSTITUTO COLOMBIANO AGROPECUARIO. 1992. Fertilización en diversos cultivos, quinta aproximación. Produmedios. Bogotá. 64p.

22. JANHUNEN, S.; PALOMÄKI, V.; HOLOPAINEN, T. 1995. Aluminium causes nutrient imbalance and structural changes in the needles of Scots pine without inducing clear root injuries. Trees. 9:134-142.

23. KAYAMA, M. 2001. Comparison of the aluminium tolerance of Miscanthus sinensis Anders. and Miscanthus sacchariflorus Bentham in hydroculture. Int. J. Plant Sci. 162:1025-1031.

24. KIDD, P.S.; PROCTOR, J. 2000. Effects of aluminium on the growth and mineral composition of Betula pendula Roth. J. Exp. Bot. 51(347):1057-1066.

25 KINRAIDE, T.B. 1993. Aluminum enhancement of plant growth in acid rooting media. A case of reciprocal alleviation of toxicity by two toxic cations. Physiol. Plant. 88:619-625.
26. KINRAIDE, T.B. 1997. Reconsidering the rhizotoxicity of hydroxyl, sulphate and fluoride complexes of aluminum. J. Exp. Bot. 48:1115-1124.

27. KOLLMEIER, M.; FELLE, H.H.; HORST, W.J. 2000. Genotypical differences in aluminum resistance of maize are expressed in the distal part of the transition zone. Is reduce basipetal auxin flow involved in inhibition of root elongation by aluminum? Plant Physiol. 122(3):945-956.

28. KONISHI, S.; MIYAMOTO, S.; TAKI, T. 1985. Stimulatory effects of aluminium on tea plants grown under low and high phosphorus supply. Soil Sci. Plant Nutr. 31:361-368.

29. LAZOF, D.B.; HOLLAND, M.J. 1999. Evaluation of the aluminum-induced root growth inhibition in isolation from low pH effects in Glycine max, Pisum sativum and Phaseolus vulgaris. Australian J. Plant Physiol. 26:147-157.

30. LLUGANY, M.; POSCHENRIEDER, C.; BARCELO, J. 1995. Monitoring of aluminium-included inhibition of root elongation in four maize cultivars differing in tolerance to $\mathrm{Al}$ and proton toxicity. Physiol. Plant. 93:265-271.

31. MA, J.F. 2005. Plant root responses to three abundant soil minerals: Silicon, aluminum and iron. Crit. Rev. Plant Sci. 24(4):267-281.

32. MARSCHNER, H. 1995. Mineral nutrition of higher plants. $2^{a}$ ed. Academic Press, Londres. p.606613.

33. MATSUMOTO, H. 1991. Biochemical mechanism of the toxicity of aluminum and the sequestration of aluminum in plant cells. En: R.J. Wright, V.C. Baligar, R.P. Murrmann, eds. Plant-Soil Interactions at Low pH. Kluwer Academic Publishers, Dordrecht, The Netherlands, p.825-838.

34. MENGEL, K.; KIRKBY, E.A. 1987. Soil as a plant nutrient medium. En: Principles of Plant Nutrition, $4^{\text {th }}$ Ed., International Potash Institute, p.56-61.

35. OSAKI, M.; WATANAD, T.; TADAMO, T. 1997. Beneficial effect of aluminium on growth of plants 
adapted to low pH soils. Soil Sci. Plant Nutr. 43:551-563.

36. PANDA, S.K.; SINGHA, L.B.; KHAN, M.H. 2003. Does aluminium phytotoxicity induce oxidative stress in greengram (Vigna radiata)?. Bulg. J. Plant Physiol. 29(1-2):77-86.

37. RAYBURN, A.L.; BIRABAR, D.P.; BULLOCK, D.G.; McMURPHY, L.M. 1993. Nuclear DNA content in F1-hybrids of maize. Heredity. 70:294-304.

38. RENGEL, Z. 1992. Disturbance of cell $\mathrm{Ca}^{2+}$ homeostasis as a primary trigger of Al toxicity syndrome. Plant Cell Environ. 15:931-938.

39. RYAN, P.R.; DiTOMASO, J.M.; KOCHIAN, L.V. 1993. Aluminium toxicity in roots: An investigation of spatial sensitivity and the role of the root cap. J. Exp. Bot. 44:437-446.

40. SÁNCHEZ, P.A. 1977. Advances in management of Oxisols and Ultisols in tropical South America. En: Proceedings of the international seminar on soil, environment, and fertility management in intensive agriculture. Tokyo, Japón. p.535-566.

41. SALINAS, J. 1988. Adaptación de plantas a toxicidades de aluminio y manganeso. En: Fertilidad de suelos: Diagnóstico y control. S.C.C.S. Bogotá. p.379-400.

42. SILVA, I.R.; SMYTH, T.J.; ISRAEL, D.W.; RAPER, C.D.; RUFTY, T.W. 2001. Magnesium ameliorates aluminum rhizotoxicity in soybean by increasing citric acid production and exudation by roots. Plant Cell Physiol. 42:546-554.

43. SILVA, I.R.; SMYTH, T.J.; MOXLEY, D.F.; CARTER, T.E.; ALLEN, N.S.; RUFTY, T.W. 2000. Aluminum accumulation at nuclei of cells in the root tip:
Fluorescence detection using lumogallion and confocal laser scanning microscopy. Plant Physiol. 123:543-552.

44. SYMEONIDIS, L.; ABOU AUDA, M.M.; YUPSANIS, T. 2003. Aluminium toxicity effects on Cucumis melo and response of diphosphonucleoside kinases. Biologia, Bratislava. 59:133-139.

45. TESFAYE, M.; TEMPLE, S.J.; ALLAN, D.L.; VANCE, C.P.; SAMAC, D.A. 2001. Overexpression of malate dehydrogenase in transgenic alfalfa enhances organic acid synthesis and confers tolerance to aluminium. Plant Physiol. 127:1836-1844.

46. Von UEXKÜLL, H.R; MUTERT, E. 1995. Global extent, development and economic impact of acid soils. En: Date, R.A.; Grundon, N.J.; Raymet, G.E.; Probert, M.E., eds. Plant-Soil Interactions at low $\mathrm{pH}$ : Principles and Management. Kluwer Acad. Publ., Dordrecht, The Netherlands, p.5-19.

47. WETZEL, J.B.; AREF, S.; BALIGAR, V.C.; RAYBURN, A.L. 1999. A lack of nuclear DNA content variability among wheat near isolines differing in aluminium response. Ann. Bot., 83:725-728.

48. YANG, Y.H.; CHEN, S.M.; ABDULLAHI, B.A.; 2001. Alleviation effect of different ratios of $\mathrm{Al}$ to $\mathrm{Ca}$ on Al toxicity for morphological growth of mungbean seedling. J. Plant Nutr. 24:573-583.

49. ZHANG, W.H.; RENGEL, Z. 1999. Aluminium induces an increase in cytoplasmic $\mathrm{Ca} 2+$ in intact wheat roots. Australian J. Plant Physiol. 26:401-409.

Recibido: Abril 15 de 2008

Aceptado: Junio 19 de 2009 